\title{
Programmed Death-Ligand 1 Expression and Its Correlation with Lymph Node Metastasis in Papillary Thyroid Carcinoma
}

Hyo Jung $\mathrm{An}^{1}$. Gyung Hyuck Ko ${ }^{2,3,4}$ Jeong-Hee Lee ${ }^{2,3,4} \cdot$ Jong Sil Lee ${ }^{2,3,4}$ Dong Chul Kim ${ }^{2,3,4} \cdot$ Jung Wook Yang ${ }^{4}$ Min Hye Kim ${ }^{4}$ Jin Pyeong Kim²,3,5 Eun Jung Jung ${ }^{2,3,6} \cdot$ Dae Hyun Song ${ }^{1,2,3}$

'Department of Pathology, Gyeongsang National University Changwon Hospital, Changwon; ${ }^{2}$ Gyeongsang National University School of Medicine, Jinju; ${ }^{3}$ Gyeongsang Institute of Health Science, Jinju; ${ }^{4}$ Department of Pathology, Gyeongsang National University Hospital, Jinju; Deparments of ${ }^{5}$ Otorhinolaryngology and ${ }^{6}$ Surgery, Gyeongsang National University Changwon Hospital, Changwon, Korea

Received: June 14, 2017

Revised: July 24, 2017

Accepted: July 25, 2017

Corresponding Author

Dae Hyun Song, MD

Department of Pathology, Gyeongsang National

University School of Medicine, 15 Jinju-daero

816beon-gil, Jinju 52727, Korea

Tel: +82-55-214-3150

Fax: +82-55-214-3174

E-mail: golgy@hanmail.net
Background: The immunotherapeutic role of programmed death-ligand 1 (PD-L1) in life expectancy in many cancers has been highlighted. However, data regarding PD-L1 expression in papillary thyroid carcinoma (PTC) are limited. In this study, we describe the PD-L1 and programmed cell death protein 1 (PD-1) expressions in PTC and analyze their correlation with lymph node (LN) metastasis. Methods: Clinicopathological data were obtained from 116 patients with PTC who were treated in Gyeongsang National University Hospital, Jinju, Korea in 2009. Tissue microarray blocks were made using representative paraffin blocks of classical PTCs excluding follicular variants. Two pathologists graded the proportion and intensity of PD-L1 and PD-1 expression in both tumor and inflammatory cells. According to their proportions, positive PTC cells were scored as negative $(0 \%)$, grade $1(1 \%-50 \%)$, and grade $2(51 \%-100 \%)$. Similarly, positive inflammatory cells were graded as negative (0\%), grade $1(1 \%-10 \%)$, and grade $2(11 \%-20 \%)$. The intensity of each protein expression was simplified as positive or negative. Results: A statistically significant correlation exists between the proportions of PD-1 and PD-L1 expression both in papillary carcinoma $(p=.001)$ and peritumoral lymphoid cells in the thyroid $(p<.001)$. In addition, the proportion of PD-L1 expression in PTC cells was closely related to metastatic LNs $(p=.036)$. Conclusions: PD-L1 is a valuable predictive marker for LN metastasis in PTC. Immunomodulating therapies that inhibit PD-L1 might be an option for patients with LN metastasis.

Key Words: Programmed death-ligand 1; Carcinoma, papillary; Thyroid; Lymph nodes; Neoplasm metastasis
The immunotherapeutic role of programmed death-ligand 1 (PD-L1) in life expectancy in many cancers has been highlighted. In particular, pembrolizumab, which is an immune checkpoint inhibitor of PD-L1, showed significantly prolonged overall survivals in non-small-cell lung cancer patients. ${ }^{1}$ PD-L1 expression has been described in other cancers, such as lymphoma, papillary renal cell carcinoma, intrahepatic cholangiocarcinoma, esophageal squamous cell carcinoma, prostatic adenocarcinoma, breast carcinoma, gastric signet ring cell carcinoma, nasopharyngeal carcinoma, lung adenocarcinoma, testicular germ cell tumor, and glioblastoma. ${ }^{2-14}$ However, data regarding PD-L1 expression in papillary thyroid carcinoma (PTC) is scarce. In this study, we describe the expressions of PD-L1 and programmed cell death protein 1 (PD1) and analyze their correlation with the clinical features of PTC.

\section{MATERIALS AND METHODS}

\section{Case selection}

By reviewing electronic clinical charts, we obtained clinicopathologic data of PTC patients treated at Gyeongsang National University Hospital, Jinju, Korea in 2009. A total of 116 patients with classical PTCs, excluding follicular or encapsulated follicular variants, were enrolled. ${ }^{15}$ Total thyroidectomy or lobectomy with lymph node (LN) dissection was performed. The tumor stage of each patient was assessed via the American Joint Committee on Cancer (AJCC) 7 th ed. Cancer Staging system. ${ }^{16}$

Two pathologists reviewed the hematoxylin and eosin-stained glass slides. This study was approved by the Institutional Review Board of Gyeongsang National University Hospital with a waiver of informed consent (GNUH-10-026). 


\section{Tissue microarray}

Surgically resected specimens were fixed overnight in buffered neutral formalin (20\%). The samples were embedded in paraffin blocks and examined. One or two representative glass slides and matched blocks were selected via microscopic review. One core of 2-mm representative tissue was collected from each paraffin block and transplanted onto new recipient tissue microarray (TMA) blocks.

\section{Immunohistochemical analysis}

Primary antibody for PD-L1 (1:200, E1L3N, Cell Signaling Technology, Danvers, MA, USA) and PD-1 (1:100, ab52587, Abcam, Cambridge, UK) was used to investigate protein expression. The immunohistochemical (IHC) method was described in our previous report. ${ }^{17}$ Fibrotic stromal area was used as negative internal control. IHC results were scored in both tumor and inflammatory cells by two pathologists. Delicate membranous or distinct cytoplasmic staining without nonspecific stromal staining was considered positive. The number of positive cells was analyzed in two categories, PTC cells and inflammatory cells. According to their proportion, PTC cells stained positive in each core of TMA block were scored as negative, grade $1(1 \%-50 \%)$, and grade $2(51 \%-100 \%)$. Similarly, positive inflammatory cells were graded as negative, grade $1(1 \%-10 \%)$, and grade $2(11 \%-$ $20 \%)$. The intensity of each protein expression was simplified as negative or positive to increase reproducibility.

\section{Statistical analysis}

The correlation of each factor was analyzed using chi-square tests. The results were considered statistically significant with pvalue less than .05. SPSS ver. 18.0 (SPSS Inc., Chicago, IL, USA) was used for all statistical analysis.

\section{RESULTS}

\section{Clinicopathological features of 116 PTC patients}

The clinicopathological features of the 116 PTC patients are summarized in Table 1. The mean age of the PTC patients was 49.5 years (range, 25 to 88 years). A total of 54 (46.6\%), three (2.6\%), and 59 (50.9\%) patients were evaluated as T stage 1, 2, and 3, respectively. LN metastasis was diagnosed in 24 patients $(20.7 \%)$.

\section{Correlation of PD-L1 and PD-1 expression in PTC cells and inflammatory cells}

The proportion of positive cells is summarized in Table 2. For
PTC cells, 39 (33.6\%) patients exhibited PD-L1 expression, and five $(8.3 \%)$ were positive for PD-1 protein (Fig. 1). Meanwhile, for inflammatory cells, seven $(6 \%)$ and six $(5.2 \%)$ patients were positive for PD-L1 and PD-1 expressions, respectively.

\section{Correlation among PD-L1 and PD-1 expressions and clinicopathological data}

The correlation between PD-L1 and PD-1 expressions is shown in Table 3. The increased proportion of PD-L1-positive PTC cells (PDL1-PTC) was significantly correlated with increased proportion of PD-1-positive PTC cells ( $\mathrm{p}=.001$ ) and PD-1-positive inflammatory cells (PD1-IC) $(\mathrm{p}<.001)$. The increased proportion of PD-L1-positive inflammatory cells was closely related with the

Table 1. Clinicopathological features

\begin{tabular}{lc}
\hline Variable & No. (\%) \\
\hline Clinical information & \\
Age, mean (range) & $49.5(25-88)$ \\
Sex (male:female) & $20: 96$ \\
Patients number with LND & $51(44.0)$ \\
Pathologic information & \\
T stage & \\
1 & $54(46.6)$ \\
2 & $3(2.6)$ \\
3 & $59(50.9)$ \\
N stage & \\
0 & $92(79.3)$ \\
1 & $24(20.7)$ \\
PD-L1 & $39(33.6)$ \\
Positive in PTC & $7(6.0)$ \\
Positive in IC & \\
PD-1 & $5(8.3)$ \\
Positive in PTC & $6(5.2)$ \\
Positive in IC & 116 \\
\hline Total &
\end{tabular}

LND, lymph node dissection; PD-L1, programmed death-ligand 1; PTC, papillary thyroid carcinoma; IC, inflammatory cells; PD-1, programmed cell death protein 1.

Table 2. Number of patients who showed PD-L1- and PD-1-positive cells

\begin{tabular}{lrr}
\hline & PD-L1 & PD-1 \\
\hline PTC $(n=116)$ & 77 & 111 \\
Negative & 34 & 3 \\
1\%-50\% positive & 5 & 2 \\
$51 \%-100 \%$ positive & & \\
IC $(n=116)$ & 109 & 110 \\
Negative & 7 & 5 \\
1\%-10\% positive & 0 & 1 \\
\hline $11 \%-20 \%$ positive &
\end{tabular}

PD-L1, programmed death-ligand 1; PD-1, programmed cell death protein 1; PTC, papillary thyroid carcinoma; IC, inflammatory cells. 
increased proportion of PD1-IC $(\mathrm{p}<.001)$.

The relationship between the collected clinicopathological data (age, sex, T stage, and N stage) and the expressions of PDL1 and PD-1 in PTC cells and inflammatory cells was analyzed. The results indicated no significant correlation, except for the proportion of PDL1-PTC and LN metastasis ( $\mathrm{p}=.036$ ) (Table 4).

\section{DISCUSSION}

In the control of T-cell immunity, positive and negative signals regulated within the $\mathrm{T}$ cells make balance between immune cell responses. ${ }^{18}$ The receptor and ligand interactions of $\mathrm{T}$ cells can support not only positive signals to improve T-cell activation but also negative signals to eliminate T cells. ${ }^{19}$ Among the negative signals, immune checkpoint proteins, PD-1 or PD-L1 are crucial to keep self-tolerance to avoid autoimmunity, through the interaction between $\mathrm{T}$ cells and antigen-presenting cells. ${ }^{20}$ Ligation of PD-1 by PD-L1 inhibits proliferation of lymphocytes and cytokine production by activated cytotoxic T cells. ${ }^{21}$ The PD-1/PD-L1 checkpoint pathway regulates not only virally infected $\mathrm{T}$ cells in the peripheral tissue $e^{21}$ but also transformed cells within the tumor microenvironment. ${ }^{22,23}$ Tumor cells co-work with immune checkpoints to avoid being destructed by host immune system.

Activated T lymphocytes normally induce PD-L1 expression

Table 3. The p-values of chi-square tests between positive proportion of PD-L1 and PD-1 at each TMA cores

\begin{tabular}{lccccc}
\hline & \multicolumn{2}{c}{ PTC } & & \multicolumn{2}{c}{ IC } \\
\cline { 2 - 3 } \cline { 5 - 6 } & PD-L1 & PD-1 & & PD-L1 & PD-1 \\
\hline PTC & & & & & \\
PD-L1 & - & .001 & & .071 & $<.001$ \\
PD-1 & .001 & - & & .126 & .172 \\
IC & & & & & \\
PD-L1 & .071 & .126 & & - & $<.001$ \\
PD-1 & $<.001$ & .172 & & $<.001$ & - \\
\hline
\end{tabular}

PD-L1, programmed death-ligand 1; PD-1, programmed cell death protein 1; TMA, tissue microarray; PTC, papillary thyroid carcinoma; IC, inflammatory cells.

Table 4. Correlation between PD-L1-positive PTC cells and metastasis to lymph nodes

\begin{tabular}{lrcc}
\hline \multirow{2}{*}{$\begin{array}{l}\text { Proportion of PD-L1 expression } \\
\text { in PTC cells (\%) }\end{array}$} & \multicolumn{3}{c}{ LN metastasis } \\
\cline { 2 - 4 } & No & Yes & Total \\
\hline 0 & 65 & 12 & 77 \\
$1-50$ & 25 & 9 & 34 \\
$51-100$ & 2 & 3 & 5 \\
Total & 92 & 24 & $\mathrm{p}=.036$ \\
\hline
\end{tabular}

PD-L1, programmed death-ligand 1; PTC, papillary thyroid carcinoma; LN, lymph node. on macrophages or host cells. Besides, there are some tumor cells that erratically express PD-L1. ${ }^{19}$ PD-L1, which is called $\mathrm{B} 7-\mathrm{H} 1$ or $\mathrm{CD} 27$, is one of the B7 gene family members expressed on the surface of the tumor cells. When effector $\mathrm{T}$ cells are activated, PD-1 is highly expressed on the regulatory T cells (Treg cells). The immune responses of Treg cells remain to be discovered completely. By producing inhibitory cytokines, they have an essential role for immune resistance in many tumors. ${ }^{20}$ Meanwhile, T cell exhaustion was first reported with chronic lymphocytic choriomeningitis virus infection of mice during which activated virusspecific $\mathrm{T}$ cells without effector function persist. ${ }^{24}$ Exhausted $\mathrm{T}$ cells overexpress multiple cell-surface inhibitory receptors, including PD-1, LAG-3, and 2B 4. ${ }^{25}$ These cells are not able to reduce PD-L1 expression, which is normally suppressed when T cells are activated. ${ }^{25}$

Tumors with PD-1 positive exhausted T cells and Treg cells may show high proportion of PD-L1-positive tumor cells. In this study, a statistically significant association existed between the expression of PD-1 and PD-L1 markers both in papillary carcinoma $(\mathrm{p}=.001)$ and lymphoid cells $(\mathrm{p}<.001)$. Moreover, the proportion of PD-L1 expression in papillary carcinoma cells was closely associated with metastatic LNs ( $\mathrm{p}=$.036). Similarly, PD-L1 was high $(\mathrm{p}=.0443)$ in patients with nodal metastases in a previous study. ${ }^{26}$ In summary, our clinical data of PTC with LN metastasis was significantly associated with PD-1/PD-L1 pathway. Although 12 out of 24 cases were negative for PD-L1 expression in PTC with metastatic LNs, the proportion of its expression and the predictive value are still meaningful. Exhausted T cells change the expression of molecules related to cell adhesion, migration, and chemotaxis during chronic viral infection. ${ }^{25}$ We assumed that PD-1-positive exhausted T cells must have altered the expression of molecules, probably modifying migratory characters in the tumor microenvironment along the metastatic cascade.

In previous studies regarding regionally metastatic differentiated thyroid carcinomas, PD-1-positive T cells and Treg cells were higher in tumor-involved LNs compared with those not involved and were related to recurrent disease. ${ }^{26}$ The association between elevated Treg cell levels and recurrent disease supports the hypothesis that Treg cells are induced by the residual metastatic tumor to avoid immune responses. ${ }^{23}$ Because tumors with high levels of infiltrating Treg cells may suppress immune responses, inhibition of the PD-1/PD-L1 pathway may improve antitumor immune responses by reducing the number of or suppressing Treg cells. $^{20}$

Our data support the existence of PD-L1 blockades in patients with thyroid carcinoma with metastatic LNs and its predictive 

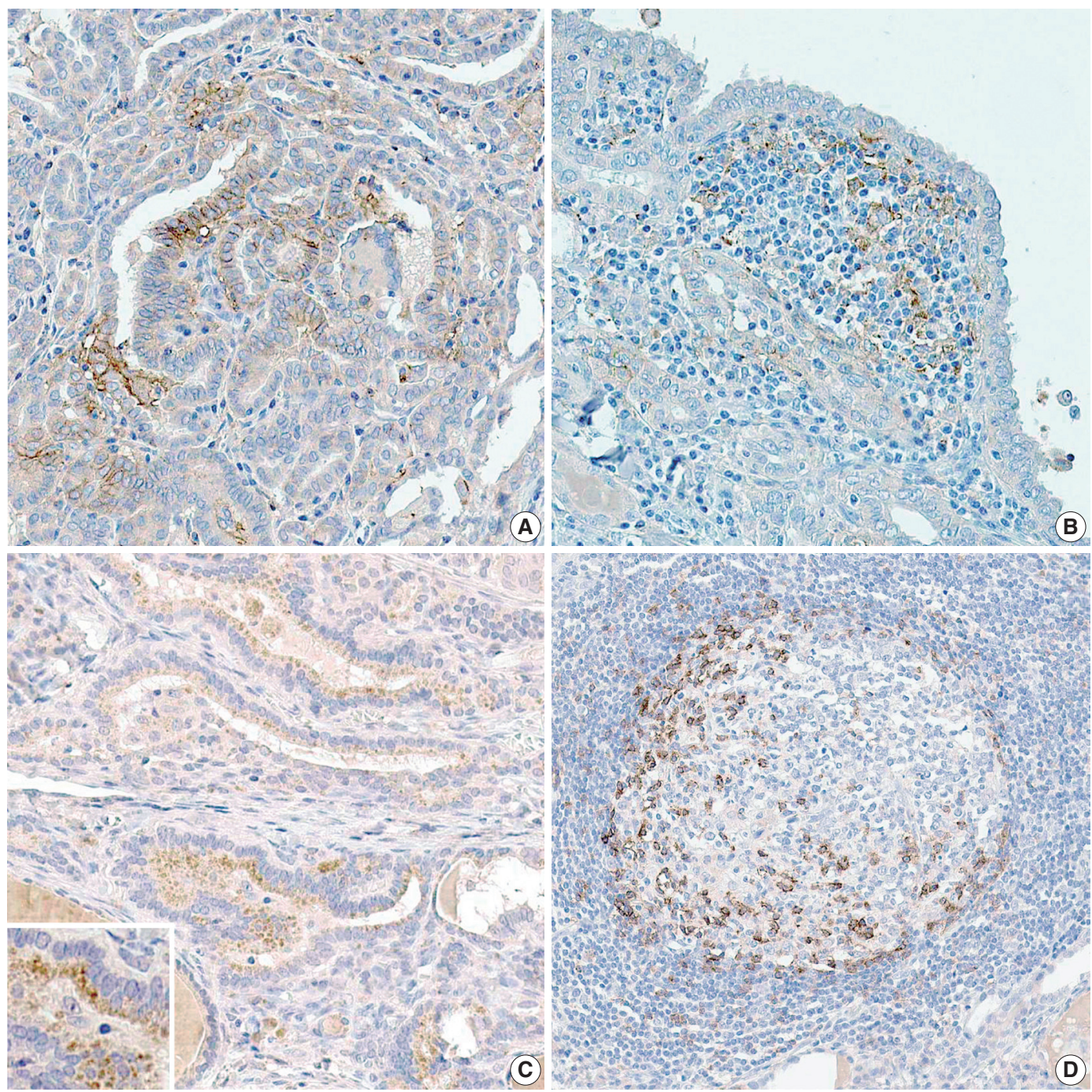

Fig. 1. (A) More than one-half of papillary carcinoma cells (PTCs) show mild programmed death-ligand 1 (PD-L1) expression in the cytoplasm. There are many PTCs with strong PD-L1 expression in cellular membrane in the center of the figure. (B) Inflammatory cells that surround PTCs exhibit PD-L1 expression. (C) Most of the PTCs reveal programmed cell death protein 1 (PD-1) expression in the perinuclear cytoplasmic area. (D) Inflammatory cells in the germinal center have reactivity to PD-1.

value as a biomarker for metastatic LNs. Immunomodulating therapies that suppress PD-L1 might be used for patients with persistent disease because regionally metastatic thyroid carcinomas are not eliminated by the host immune system. The prognostic role of PD-L1 expression associated with LN metastasis was elucidated in this study.

\section{Conflicts of Interest}

No potential conflict of interest relevant to this article was reported.

\section{Acknowledgments}

This work was funded by the research promotion program of Gyeongsang National University, 2016.

\section{REFERENCES}

1. Herbst RS, Baas P, Kim DW, et al. Pembrolizumab versus docetaxel for previously treated, PD-L1-positive, advanced non-small-cell lung cancer (KEYNOTE-010): a randomised controlled trial. Lancet 2016; 387: 1540-50.

2. Juárez-Salcedo LM, Sandoval-Sus J, Sokol L, Chavez JC, Dalia S. The role of anti-PD-1 and anti-PD-L1 agents in the treatment of diffuse large B-cell lymphoma: The future is now. Crit Rev Oncol Hematol 
2017; 113: 52-62.

3. Motoshima T, Komohara Y, Ma C, et al. PD-L1 expression in papillary renal cell carcinoma. BMC Urol 2017; 17: 8.

4. Fontugne J, Augustin J, Pujals A, et al. PD-L1 expression in perihilar and intrahepatic cholangiocarcinoma. Oncotarget 2017; 8: 2464451.

5. Jiang Y, Lo AW, Wong A, et al. Prognostic significance of tumor-infiltrating immune cells and PD-L1 expression in esophageal squamous cell carcinoma. Oncotarget 2017; 8: 30175-89.

6. Ness N, Andersen S, Khanehkenari MR, et al. The prognostic role of immune checkpoint markers programmed cell death protein 1 (PD-1) and programmed death ligand 1 (PD-L1) in a large, multicenter prostate cancer cohort. Oncotarget 2017; 8: 26789-801.

7. Park IH, Yang HN, Lee KJ, et al. Tumor-derived IL-18 induces PD-1 expression on immunosuppressive NK cells in triple-negative breast cancer. Oncotarget 2017; 8: 32722-30.

8. Jin S, Xu B, Yu L, et al. The PD-1, PD-L1 expression and CD3+ T cell infiltration in relation to outcome in advanced gastric signet-ring cell carcinoma, representing a potential biomarker for immunotherapy. Oncotarget 2017; 8: 38850-62.

9. Gravelle P, Burroni B, Péricart S, et al. Mechanisms of PD-1/PD-L1 expression and prognostic relevance in non-Hodgkin lymphoma: a summary of immunohistochemical studies. Oncotarget 2017; 8: 44960-75.

10. Zhou Y, Miao J, Wu H, et al. PD-1 and PD-L1 expression in 132 recurrent nasopharyngeal carcinoma: the correlation with anemia and outcomes. Oncotarget 2017; 8: 51210-23.

11. Chen N, Fang W, Lin Z, et al. KRAS mutation-induced upregulation of PD-L1 mediates immune escape in human lung adenocarcinoma. Cancer Immunol Immunother 2017; 66: 1175-87.

12. Chovanec M, Cierna Z, Miskovska V, et al. Prognostic role of programmed-death ligand 1 (PD-L1) expressing tumor infiltrating lymphocytes in testicular germ cell tumors. Oncotarget 2017; 8: 21794-805.

13. Botti G, Collina F, Scognamiglio G, et al. Programmed death ligand 1 (PD-L1) tumor expression is associated with a better prognosis and diabetic disease in triple negative breast cancer patients. Int J Mol Sci 2017; 18: E459.

14. Han J, Hong Y, Lee YS. PD-L1 expression and combined status of PD-L1/PD-1-positive tumor infiltrating mononuclear cell density predict prognosis in glioblastoma patients. J Pathol Transl Med 2017; 51: 40-8

15. Nikiforov YE, Seethala RR, Tallini G, et al. Nomenclature revision for encapsulated follicular variant of papillary thyroid carcinoma: a paradigm shift to reduce overtreatment of indolent tumors. JAMA Oncol 2016; 2: 1023-9.

16. Nixon IJ, Wang LY, Migliacci JC, et al. An international multi-institutional validation of age 55 years as a cutoff for risk stratification in the AJCC/UICC staging system for well-differentiated thyroid cancer. Thyroid 2016; 26: 373-80.

17. Song $\mathrm{DH}, \mathrm{Ko} \mathrm{GH}$, Lee JH, et al. Myoferlin expression in non-small cell lung cancer: Prognostic role and correlation with VEGFR-2 expression. Oncol Lett 2016; 11: 998-1006.

18. Chen L. Co-inhibitory molecules of the B7-CD28 family in the control of T-cell immunity. Nat Rev Immunol 2004; 4: 336-47.

19. Thompson RH, Dong H, Kwon ED. Implications of B7-H1 expression in clear cell carcinoma of the kidney for prognostication and therapy. Clin Cancer Res 2007; 13(2 Pt 2): 709s-15s.

20. Pardoll DM. The blockade of immune checkpoints in cancer immunotherapy. Nat Rev Cancer 2012; 12: 252-64.

21. Freeman GJ, Long AJ, Iwai Y, et al. Engagement of the PD-1 immunoinhibitory receptor by a novel B7 family member leads to negative regulation of lymphocyte activation. J Exp Med 2000; 192: 1027-34.

22. Dong H, Strome SE, Salomao DR, et al. Tumor-associated B7-H1 promotes T-cell apoptosis: a potential mechanism of immune evasion. Nat Med 2002; 8: 793-800.

23. French JD, Kotnis GR, Said S, et al. Programmed death-1+ T cells and regulatory $\mathrm{T}$ cells are enriched in tumor-involved lymph nodes and associated with aggressive features in papillary thyroid cancer. J Clin Endocrinol Metab 2012; 97: E934-43.

24. Zajac AJ, Blattman JN, Murali-Krishna K, et al. Viral immune evasion due to persistence of activated $\mathrm{T}$ cells without effector function. J Exp Med 1998; 188: 2205-13.

25. Wherry EJ, Ha SJ, Kaech SM, et al. Molecular signature of CD8+ T cell exhaustion during chronic viral infection. Immunity 2007; 27: 670-84.

26. Bastman JJ, Serracino HS, Zhu Y, et al. Tumor-infiltrating T cells and the PD-1 checkpoint pathway in advanced differentiated and anaplastic thyroid cancer. J Clin Endocrinol Metab 2016; 101: 286373. 